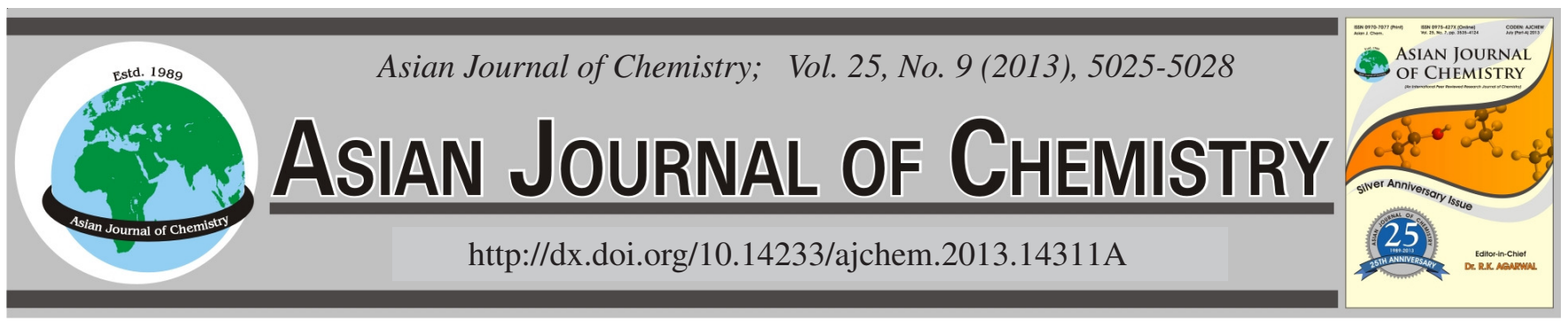

\title{
Ammonium Persulfate-Induced Graft Copolymer Based on Carboxymethyl Cellulose-g-PMAAm-co-PNIPAAm
}

Fatemeh Soleimani ${ }^{*}$, Hossein Sadeghi, Hadis Shahsavari, Arezou Soleimani and Fatemeh Sadeghi

Department of Chemistry, Science Faculty, Islamic Azad University, Arak Branch, Arak, Iran

*Corresponding author: E-mail: fatisoleymani@yahoo.com

(Received: 13 July 2012;

Accepted: 18 March 2013)

AJC-13128

In this study, a novel graft copolymer based on sodium carboxymethyl celloluse via a free radical polymerization method is synthesized. The graft copolymerization reaction was carried out in a homogeneous medium and by reaction of N-isopropylacrylamide and methacrylamide with carboxymethyl cellulose backbones. Evidence of grafting was obtained by comparing FTIR and TGA analysis of carboxymethyl cellulose and the graft copolymer as well as solubility characteristics of the products.

Key Words: Carboxymethyl cellulose, Grafting, Methacrylamide, N-Isopropylacrylamide.

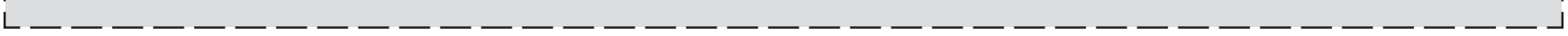

\section{INTRODUCTION}

Graft copolymerization of hydrophilic and hydrophobic vinyl monomers is a well-known technique employed by polymer chemists for significantly modifying the chemical and physical properties of the synthetic or natural starting materials with minimum degradation of the original properties ${ }^{1-4}$. Graft copolymers are prepared by first generating free radicals on the polysaccharide backbone and then allowing these radicals to serve as macroinitiators for the vinyl monomers. These biodegradable and low cost graft copolymers, with new properties can be used in many applications such as textiles, paper industry, agriculture, medical treatment, in petroleum industry as flocculants and thickening agents ${ }^{5-7}$ and also development of selective permeable membranes ${ }^{8}$, sorption agents $^{9}$ and in fabrication of drug delivery systems ${ }^{10,11}$. Grafting can be performed using free radical initiators, redox systems or photochemical process persulfate in its state (for example APS, KPS) is a versatile oxidizing agent that through various redox reactions with many different organic substrates can create free radicals capable of initiating vinyl polymerizations ${ }^{1}$. Ammonium persulfate has been widely used by many investigators for initiating graft copolymerization of vinyl monomers onto various natural and synthetic polymers. The literature survey, however, reveals that few of the modifications deal with chemical grafting of a pre-modified polysaccharide such as carboxymethyl cellulose. Ammonium persulfate-initiated grafting of vinyl monomers such as methyl acrylate, ethyl acrylate and ethyl methacrylate ${ }^{7,8}$, AN/methyl methacrylate mixture $^{9}$, acrylamide (AAm) ${ }^{10,11}$ and 4-vinylpyridine ${ }^{12,13}$ onto carboxymethyl cellulose has been reported. using different initiators, acrylic acid has also been photografted onto carboxymethyl cellulose. In addition, N-vinyl-2-pyrrolidone and its mixture with AA has been graft copolymerized onto mixtures of carboxymethyl cellulose/hydroxyethylcellulose to prepare cellulosic membrane with special biological effects. However, to the best of our knowledge, no report has been published on the optimization simultaneously of methacrylamide and $\mathrm{N}$-isopropylacrylamide grafted onto carboxymethyl cellulose using APS-saccharide initiating system. As a part of our research program on polysaccharide modification, herewith we report the optimized APS-induced synthesis of CMC$g$-poly(MAAm-co-NIPAAm) under an inert atmosphere ${ }^{2}$.

\section{EXPERIMENTAL}

The polysaccharide, carboxymethylcellulose (CMC, degree of substitution 0.63, from Condinson Co., Denmark) was of analytical grade and was used as received. N-Isopropylacrylamide (NIPAAm, Merck) and methacrylamide (MAAm, Merck) were used as received. Ammonium persulfate (APS, Merck) was used without purification.

Graft polymerization: A general procedure was conducted as follows: carboxymethyl cellulose solution was prepared in a $1 \mathrm{~L}$ reactor equipped with mechanical stirrer and gas inlet. Carboxymethyl cellulose was dissolved in degassed distillated water. In general, $0.50 \mathrm{~g}$ of carboxymethyl cellulose was dissolved in $30.0 \mathrm{~mL}$ of distillated degassed water. The reactor was placed in a water bath preset at $60{ }^{\circ} \mathrm{C}$. Then $0.10 \mathrm{~g}$ of 
ammonium persulfate (dissolved in $5 \mathrm{~mL}$ water) as an initiator was added to carboxymethyl cellulose solution and was allowed to stir for $10 \mathrm{~min}$ at $60{ }^{\circ} \mathrm{C}$. After adding initiator, simultaneously variable amounts of MAAm and NIPAAm were added to the carboxymethyl cellulose solution. After $1 \mathrm{~h}$, the reaction product was allowed to cool to ambient temperature and methanol $(500 \mathrm{~mL})$ was added to the product. After complete dewatering for $24 \mathrm{~h}$, the product was filtered, washed with fresh methanol $(2 \mathrm{~mL} \times 50 \mathrm{~mL})$ and dried at $50^{\circ} \mathrm{C}$.

Infrared analysis: The samples were crushed with $\mathrm{KBr}$ to make pellets. Spectra were taken on an ABB Bomem MB-100 FTIR spectrophotometer.

Thermal analysis: Thermogravimetric analyses were performed on a Universal V4.1D TA Instruments (SDT Q600) with 8-10 mg samples on a platinum pan under nitrogen atmosphere. Experiments were performed at a heating rate of $10{ }^{\circ} \mathrm{C} / \mathrm{min}$ until $570{ }^{\circ} \mathrm{C}$.

\section{RESULTS AND DISCUSSION}

Grafting mechanism and rate: PMAAm and PNIPAAm monomers was simultaneously grafted onto CMC in a homogenous medium using APS as a radical initiator under an inert atmosphere.

The mechanism of co polymerization of PMAAm and PNIPAAm monomers onto CMC in the presence of thermal initiator is shown in Scheme-I. In the first step, the persulfate initiator is decomposed under heating to generate sulfate anion-radical (Scheme-I). The radical abstracts hydrogen from the hydroxyl group of the polysaccharide substrate to form alkoxy radicals on the substrate. So, this persulfate-saccharide redox system is resulted in active centers on the substrate to radically initiate polymerization of PMAAc and PNIPAAm monomers onto CMC backbones led to a graft copolymer.

The rate of grafting $\left(\mathrm{R}_{\mathrm{g}}\right)$ may be evaluated as measures of the rate of monomers disappearance by using the following $\operatorname{expression}^{14}$.

$$
\mathrm{R}_{\mathrm{g}}\left(\mathrm{mol} \mathrm{L}^{-1} \mathrm{~s}^{-1}\right)=\frac{1000 \mathrm{~W}_{3}}{\mathrm{MTV}}
$$

$\mathrm{M}\left(\mathrm{g} \mathrm{mol}^{-1}\right)$ is the molecular weight of the monomers. T and $\mathrm{V}$ stand for total reaction time $(\mathrm{s})$ and total volume $(\mathrm{mL})$ of the reaction mixture.

Overall activation energy of grafting $\left(E_{a}\right)$ may also be estimated from the temperature data through plotting $\ln \mathrm{R}_{\mathrm{g}}$ versus $1 / \mathrm{T}\left(\mathrm{K}^{-1}\right)$ for the initial portion of the data of the temperature series given in text. The slope of this Arrhenius plot (Fig. 1) resulted in a rough estimation of $E_{a}$ of grafting using the relationship slope $=-E_{a} / R$; where $R$ is the universal gas constant. Therefore, $\mathrm{E}_{\mathrm{a}}$ was found to be $36.60 \mathrm{~kJ} / \mathrm{mol}(8.75$ $\mathrm{kcal} / \mathrm{mol}$ ).

Evidence of grafting: For identification of the copolymer formation, infrared spectroscopy was used. Fig. 2 shows the IR spectroscopy of CMC- $g$-poly(MAAm-co-NIPAAm) copolymer. The copolymer product comprises a CMC backbone with side chains that carry carboxamide functional groups that are evidenced by peaks at $1679 \mathrm{~cm}^{-1}$. In fact, In the spectrum of the copolymer (Fig. 2b), new peaks are appeared at 3203 and $1679 \mathrm{~cm}^{-1}$ that may be attributed to amide NH stretching, asymmetric and symmetric amide NH bending, respectively.

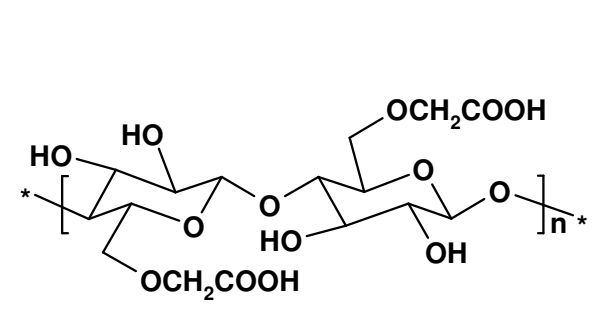

$(\mathrm{CMC}=\mathrm{ROH})$

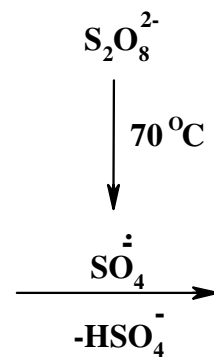

RÖ

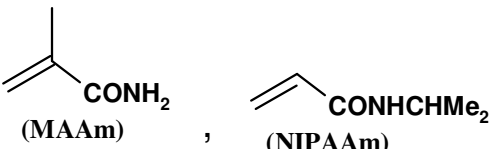

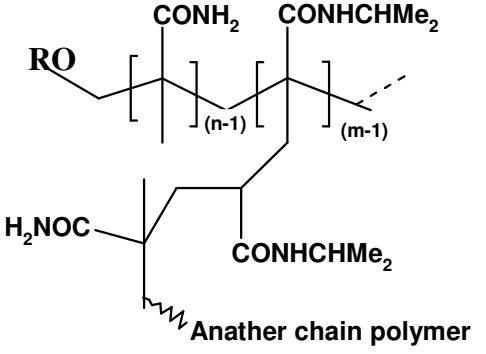
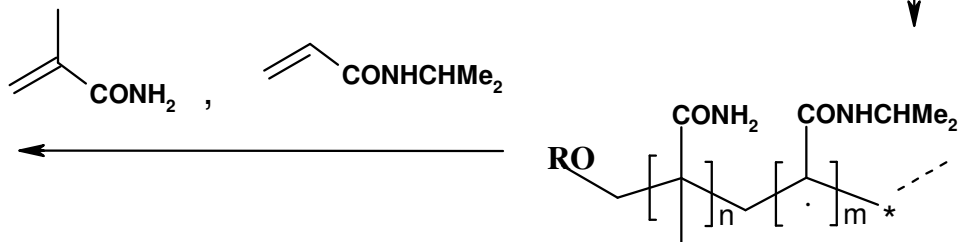

CMC-g-poly(MMAm-co-NIPAAm) graft copolymer

Scheme-I: A brief proposed mechanism for APS-induced grafting of poly(MAAm-co-NIPAAm) onto CMC 


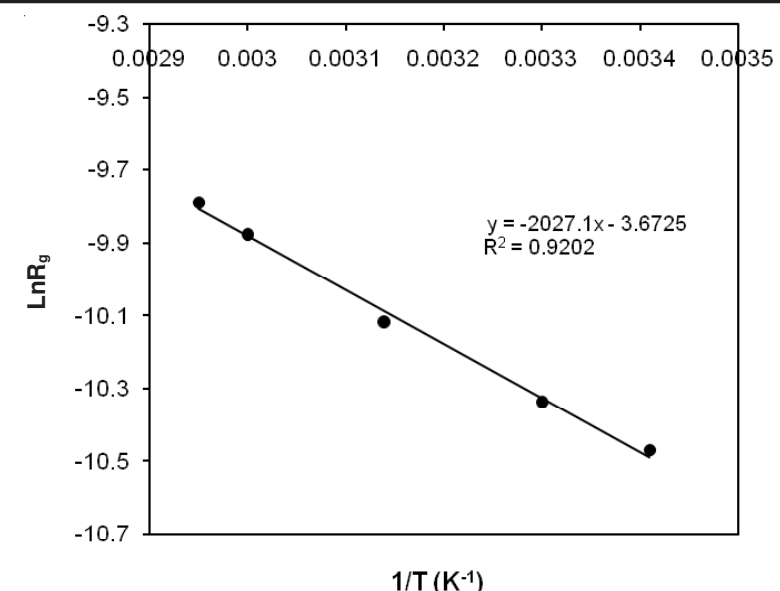

Fig. 1. Plot of $\ln \mathrm{R}_{\mathrm{g}}-1 / \mathrm{T}$ for estimating the activation energy of the graft polymerization reaction

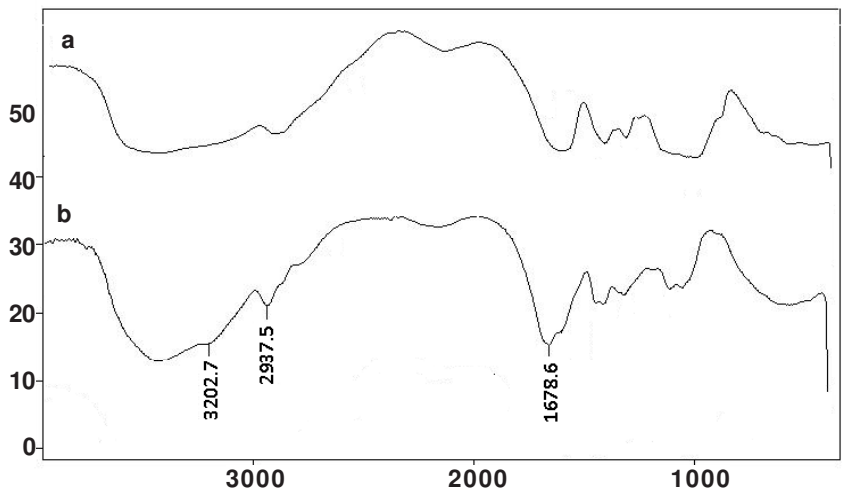

Fig. 2. FTIR spectra of pure CMC (a), CMC-g-poly(MAAm-co-NIPAAm) copolymer (b)

Thermogravimetric behaviour: Thermogravimetric analysis was employed to thermally characterize the CMC- $g$ poly(MAAm-co-NIPAAm) copolymer in comparison with the intact CMC (Fig. 3). The thermal stability of the grafted CMC is improved as is obvious from the TGA curve. TGA of pure CMC (Fig. 3a) shows a weight loss in two distinct stages. The first stage ranges between 10 and $130{ }^{\circ} \mathrm{C}$ and shows $\mathrm{ca} .17 \%$ loss in weight. This may correspond to the loss of adsorbed and bound water. No such inflexion was observed in the TGA curve of CMC- $g$-poly(MAAm-co-NIPAAm) copolymer (Fig. 3b).

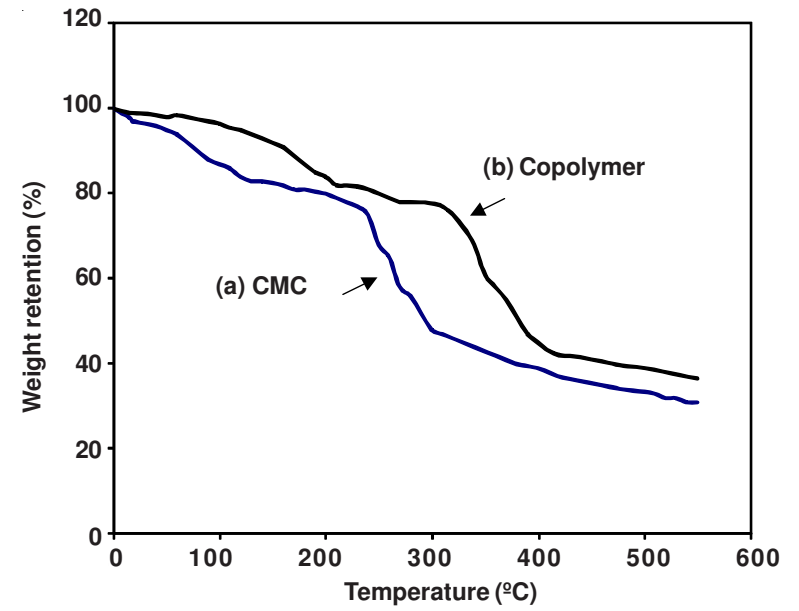

Fig. 3. TGA of pure CMC (a) and CMC-g-poly(MAAm-co-NIPAAm) copolymer (b)
This indicated that the grafted copolymers were resistant to moisture absorption. The second stage of weight loss starts at $230{ }^{\circ} \mathrm{C}$ and continues up to $300{ }^{\circ} \mathrm{C}$ during which there was $52 \%$ weight loss due to the degradation of CMC. In general, degradation of native $\mathrm{CMC}$ is faster than that of CMC-gpoly(MAAm-co-NIPAAm) copolymer. $\mathrm{Ca}$. $60 \%$ weight loss takes place in the temperature range of $220-370{ }^{\circ} \mathrm{C}$ for $\mathrm{CMC}$. In the CMC-g-poly(MAAm-co-NIPAAm) copolymer, a residual weight of $77 \%$ was observed at $310^{\circ} \mathrm{C}$. The appearance of these stages indicates the structure of CMC backbones has been changed, which might be due to the grafting of poly(MAAm-co-NIPAAm) chains. In general, the copolymer had lower weight loss than CMC. This means that the grafting of CMC increases the thermal stability of CMC in some extent $^{13,15}$.

Solubility test: To obtain an additional evidence of grafting, solubility difference between the grafted and the non-grafted polymer was used carboxymethylcellulose and poly(MAAm-co-NIPAAm) are soluble in water and DMF, respectively. When a reaction product was extracted with DMF and alternatively with water for $24 \mathrm{~h}$, an insoluble solid still remained. A physical mixture of CMC and poly(MAAm-coNIPAAm) was treated in the same way and was found to dissolve completely. Therefore, it is obvious that the resulted graft copolymer was not a simple physical mixture, but some chemical bonds must exist between the CMC substrate and poly(MAAm-co-NIPAAm) macromolecules. In addition to the formation of graft copolymers, crosslinking between the chains of CMC may also take place ${ }^{16}$. This was evident by the reaction between carboxymethylcellulose and the initiator, in the absence of monomers, giving a product with reduced solubility (unpublished data).

Gravimetric analysis: The graft copolymerization reaction was monitored gravimetrically. Increase in the mass of carboxymethylcellulose, after extraction of homopolymers, was taken as evidence for grafting. This weight gain in CMC forms the basis for the determination of the grafting parameters ${ }^{17}$.

\section{Conclusion}

The monomers, methacrylamide (MAAm) and Nisopropylacrylamide (NIPAAm), can be easily graft copolymerized onto CMC polysaccharide using APS as an initiator in aqueous medium under an inert atmosphere. In order to prove that MAAm and NIPAAm monomers were grafted, solubility test, FTIR spectroscopy, TGA analysis and gravimetric analysis were used. Empirical polymerization rate showed a first-order dependence on the monomers concentration and a half-order dependence on the initiator concentration. According to the slope of $\ln \mathrm{R}_{\mathrm{g}}$ versus $1 / \mathrm{T}$, the overall activation energy for graft copolymerization reaction was estimated to be 36.60 $\mathrm{kJ} / \mathrm{mol}$.

\section{REFERENCES}

1. F.L. Buchholz and A.T. Graham, Modern Superabsorbent Polymer Technology, Wiley, New York (1997).

2. V.D. Athawale and V. Lele, Carbohydr. Polym., 35, 21 (1998).

3. D. Castal, A. Ricard and R. Audebert, J. Appl. Polym. Sci., 39, 11 (1990).

4. P.J. Flory, Principles of Polymer Chemistry, Cornell University Press, Ithaca, New York (1953). 
5. L.H. Gan, G.R. Deen, Y.Y. Gan and K.C. Tam, Eur. Polym. J., 37, 1473 (2001).

6. S. Hua and A. Wang, Carbohydr. Polym., 75, 79 (2009).

7. J. Kost, Encyclopedia of Controlled Drug Delivery, Intelligent Drug Delivery Systems, Wiley, New York, Vol. 1 (1999).

8. W.F. Lee and W.Y. Yuan, J. Appl. Polym. Sci., 77, 1760 (2000).

9. G.R. Mahdavinia, A. Pourjavadi, H. Hosseinzadeh and M.J. Zohuriaan, Eur. Polym. J., 40, 1399 (2004).

10. N.A. Peppas and A.G. Mikes, Hydrogels in Medicine and Pharmacy, CRC Press, Boca Raton, Florida (1986).

11. L.B. Peppas and R.S. Harland, Absorbent Polymer Technology, Elsevier, Amsterdam (1994).
12. R. Po, J. Macromol. Sci.-Rev. Macromol. Chem. Phys., C34, 607 (1994).

13. A. Pourjavadi, A.M. Harzandi and H. Hosseinzadeh, Eur. Polym. J., 40, 1363 (2004).

14. J. Chen and Y. Zhao, J. Appl. Polym. Sci., 75, 808 (2000).

15. A. Pourjavadi and M. Kurdtabar, Eur. Polym. J., 43, 877 (2007).

16. M.J. Zohuriaan-Mehr and A. Pourjavadi, J. Polym. Mater., 20, 113 (2003).

17. H. Yan, A. Saiani, J.E. Gough and A.F. Miller, Biomacromolecules, 7, 2776 (2006). 